\title{
MORBIDADE ENTRE A PÓS-BIÓPSIA DE LINFONODO SENTINELA E A DISSECÇÃO AXILAR NO CÂNCER DE MAMA
}

\author{
Beatriz Pifano Soares Ferreira, Mônica Duarte Pimentel, luiz Cláudio dos Santos, Walace di Flora, Helenice Gobbi* \\ Trabalho realizado na Universidade Federal de Minas Gerais, Belo Horizonte, MG
}

\author{
*Correspondência: \\ UFMG \\ Departamento de Anatomia \\ Patológica \\ Av. Alfredo Balena, 190 . \\ 30 andar \\ CEP: 30130-100 - Belo \\ Horizonte, MG \\ Tel: 34099118; Fax: \\ 34099664 \\ helenicegobbi@gmail.com
}

\begin{abstract}
RESUMO
ОвJetivo. O objetivo deste estudo foi avaliar a morbidade cirúrgica pós-biópsia de linfonodo sentinela (BLS) ou dissecção axilar com (DA-NP) e sem preservação do nervo intercostobraquial (DA-NS).

Métodos. Fez-se estudo coorte prospectivo com 108 pacientes divididas em três grupos: BLS $(n=35)$, DA-NP $(n=36)$ e DA-NS ( $n=37)$. Foram avaliadas ocorrência de déficit sensorial, dor, linfedema, seroma e infecção no membro superior homolateral à cirurgia. Monofilamentos de Semmes-Weinstein foram usados para avaliar o déficit sensorial, perimetria braquial foi feita para avaliação da presença de linfedema e aplicado questionário de dor. Para análise estatística foram utilizados os testes ANOVA e Kruskal-Wallis. Foi feita análise bivariada e multivariada.

Resultados. Pelo menos uma complicação pós-cirúrgica, imediata ou tardia, ocorreu em 45/108 (41,7\%) pacientes avaliadas. A complicação mais comum foi dor. Houve diferença estatisticamente significante entre os três grupos somente quanto ao déficit sensorial $(p=0,04)$. Dor, linfedema e déficit sensorial ocorreram com maior freqüência no grupo DA-NS. As pacientes dos grupos BLS e DA-NP não apresentaram diferenças estatisticamente significantes para nenhuma das variáveis analisadas. A pesquisa com os monofilamentos mostrou sensibilidade cutânea preservada em 28/35 pacientes do grupo BLS, em 25/36 pacientes do grupo DA-NP e em 10/37 pacientes do grupo DA-NS ( $p<0,001$ ).

Conclusão. A secção do nervo está relacionada a maior déficit sensorial havendo diferença estatisticamente significante entre os três grupos, o que não demonstrou ser significante com os demais critérios avaliados dentre os grupos analisados.

UnITERMos: Morbidade. Câncer de mama. Biópsia de linfonodo sentinela. Dissecção axilar. Preservação. Nervo intercostobraquial.
\end{abstract}

\section{INTRODUÇÃo}

O estadiamento axilar é importante etapa no tratamento do câncer de mama. A dissecção axilar (DA) foi durante anos técnica padrão para a maioria dos casos, e ainda é muito útil em grande número de pacientes afetadas pelo câncer de mama. O status axilar é fator prognóstico potente e um dos mais importantes para predizer recorrência e sobrevida!.

A abordagem cirúrgica da axila no tratamento do câncer de mama mudou mais recentemente e o conceito de conservação, aplicado anteriormente ao tratamento cirúrgico da mama com o desenvolvimento da quadrantectomia, agora também envolve os linfonodos axilares'. No final dos anos 90, a introdução da biópsia do linfonodo sentinela (BLS) revolucionou a cirurgia para estadiamento axilar por câncer de mama. Resultados de vários estudos mostraram alto valor preditivo do não-acometimento axilar nos casos em que a avaliação do linfonodo sentinela não evidenciou metástase. A BLS está associada à menor morbidade, melhor preservação da função do braço e melhor qualidade de vida das pacientes comparadas àquelas com DA².

No entanto, no Brasil, como ainda predomina diagnóstico de casos mais avançados, cirurgias mais extensas e DA ainda são muito utilizados. Em algumas mulheres, tais procedimentos estão associados à ocorrência de complicações a curto e longo prazos. As mais comuns são hemorragias, infecções, seromas, linfedema do membro superior, dor crônica, parestesia devido à lesão do nervo intercostobraquial, redução da amplitude de movimento do ombro e fraqueza homolaterais à cirurgia. Estas complicações interferem no dia-a-dia e na qualidade de vida das pacientes ${ }^{3-5}$.

O nervo sensitivo intercostobraquial (ICB) origina-se do ramo lateral do segundo nervo intercostal e é responsável pela sensibilidade cutânea da axila, região medial e póstero-superior do braço ${ }^{6}$. Sua lesão pode ocorrer durante DA e se manifesta, na maioria das pacientes, como anestesia ou hipoestesia, podendo ocorrer hiperestesia em alguns $\operatorname{casos}^{7}$. Poucos estudos na literatura abordaram a preservação cirúrgica do nervo ICB durante o DA ${ }^{3,5,7,8}$. Em nosso serviço, avaliamos a sensibilidade cutânea em mulheres que se submeteram ao DA com e sem preservação do nervo ICB e observamos que quando o nervo é preservado durante a abordagem axilar, há melhora significativa do déficit sensorial e menor morbidade 5 . A técnica cirúrgica de preservação não prolonga o tempo de cirurgia e não traz riscos à paciente ${ }^{3,5,7,8}$.

Embora estudos internacionais e brasileiros tenham demonstrado menor morbidade da BLS em relação ao DA, estes estudos não comparam a BLS e o DA com preservação do nervo ICB. Dando 
FerrelRA BPS et AL.

continuidade a nossa linha de pesquisa, definimos como objetivo do presente trabalho comparar a morbidade pós-cirúrgica de pacientes submetidas BLS com aquelas submetidas a DA com e sem preservação do nervo ICB.

\section{Métodos}

Este estudo clínico de coorte prospectivo foi realizado no serviço de mastologia da Maternidade Odete Valadares, da Fundação Hospitalar do Estado de Minas Gerais, Belo Horizonte. Foram selecionadas 108 pacientes com diagnóstico de câncer de mama, submetidas a cirurgia conservadora ou mastectomia com DA ou BLS no período de março de 2005 a junho de 2006.

As pacientes foram divididas em três grupos de acordo com a abordagem axilar. Grupo I: 35 pacientes submetidas a biópsia de linfonodo sentinela (BLS); Grupo II: 36 pacientes submetidas a dissecção axilar com nervo intercostobraquial preservado (DA-NP); Grupo III: 37 pacientes submetidas a dissecção axilar com nervo intercostobraquial seccionado (DA-NS).

Todas as cirurgias foram realizadas pela mesma equipe médica. Os critérios para a não preservação do nervo foram acometimento neoplásico linfonodal maciço, próximo à veia axilar; acometimento linfonodal na trajetória do nervo intercostobraquial; dificuldade técnica para a preservação do nervo durante a cirurgia.

Foram incluídas no estudo apenas pacientes que não eram diabéticas, sem lesões ou neuropatias periféricas, pacientes que não foram submetidas a cirurgia bilateral e a reconstrução imediata e as que não apresentavam linfedema previamente à cirurgia.

Foram excluídas do estudo pacientes que não retornaram ao ambulatório de mastologia para avaliação pós-operatória na data prevista.

O projeto foi aprovado pelos Comitês de Ética em Pesquisa da Maternidade Odete Valadares, da Fundação Hospitalar do Estado de Minas Gerais e da Universidade Federal de Minas Gerais.

As pacientes eram submetidas um ano após a cirurgia a avaliação da sensibilidade cutânea, a medida da perimetria braquial e respondiam a questionário específico de McGill para avaliação de dor adaptado por Wallace et al., $1996^{9}$

Dados referentes à idade, data, tipo de cirurgia, estado civil, nível de instrução, lateralidade, estadiamento clínico TNM, status menopausal, estadio, número de linfonodos dissecados, dados do exame anatomopatológico, número de linfonodos acometidos, preservação ou não do nervo intercostobraquial, presença ou ausência de seroma e infecção pós-operatória foram obtidos a partir dos prontuários médicos das pacientes selecionadas e que aceitaram participar do estudo. Os dados foram incluídos em protocolo de estudo desenvolvido para este trabalho.

As pacientes incluídas neste estudo foram entrevistadas pela mesma examinadora que preencheu o questionário espećifico de dor adaptado de McGill 9 .

Havendo queixa de dor, esta foi caracterizada pela intensidade, tempo de início após a cirurgia, local, tipo, duração, se a dor a fazia sentir-se incapaz para realizar atividades habituais. Anotava-se se a paciente fez uso de medicação analgésica, de medicação sistêmica, e se fez quimioterapia, radioterapia e/ou hormonioterapia. A avaliação clínica e exame físico incluíram a inspeção estática para pesquisar alterações cutâneas e perimetria braquial bilateral, medindo-se 5, 10, e $15 \mathrm{~cm}$ acima e abaixo da prega cubital.

Para a avaliação da sensibilidade cutânea, foram usados os monofilamentos de Semmes-Weinstein ou estesiômetro. A calibração de cada monofilamento, ou seja, sua espessura e força necessária para dobrá-lo, é fornecida pelo fabricante (SORRI, Bauru SP).

Este teste de sensibilidade utiliza seis monofilamentos de nylon número 612 , de $38 \mathrm{~mm}$ de comprimento e de diâmetros diferentes. Cada monofilamento é fixado a uma haste, em ângulo de $90^{\circ} \mathrm{e}$ corresponde a um nível funcional representado por uma cor e peso em gramas. A técnica de aplicação do teste seguiu as recomendações do Guia de Controle da Hanseníase do Ministério da Saúde ${ }^{10}$. O teste começava com o monofilamento mais leve e pedia-se à paciente para responder "sim" quando sentisse o toque do filamento. Na ausência de resposta, continuava-se com o próximo filamento mais pesado e assim sucessivamente.

A avaliação foi realizada na face medial superior do braço, correspondente ao dermátomo do nervo intercostobraquial, homolateral e contralateral à cirurgia. Era solicitado à paciente para deitar em decúbito dorsal, com as mãos colocadas atrás da cabeça e com os olhos fechados. Para realizar o teste, o cabo do instrumento era segurado de modo que o filamento de nylon ficasse perpendicular à superfície da pele, a uma distância de mais de $2 \mathrm{~cm}$. A pressão na pele era feita até obter a curvatura do filamento e sem permitir que o mesmo deslizasse sobre a pele.

Os resultados foram interpretados a partir das respostas das pacientes aos estímulos provocados, variando de sensibilidade normal à perda completa da sensibilidade protetora e da pressão profunda.

Considera-se grau zero de incapacidade a presença de resposta positiva aos filamentos $0,05 \mathrm{~g}$ (cor verde) ou $0,2 \mathrm{~g}$ (cor azul) ou ainda $2 \mathrm{~g}$ (cor violeta). Considera-se grau I de incapacidade a presença de resposta positiva aos filamentos $4 \mathrm{~g}$ (cor vermelha), I0g (cor laranja), $300 \mathrm{~g}$ (cor vermelha magenta) ou ainda ausência de resposta ao filamento $300 \mathrm{~g}$ (cor vermelha magenta).

\section{Análise estatística}

Foram utilizados os testes ANOVA e Kruskal-Wallis e realizadas análise univariada para descrever o perfil de morbidade do grupo e freqüência das demais variáveis. Análise bivariada foi realizada e nas que se obteve associação estatisticamente significante foi feita análise multivariada. Foi considerado estatisticamente significante o valor de $p<0,05$. Para análise estatística dos resultados foram utilizados os programas EPI INFO 6.04 e SPSSI2.

\section{Resultados}

No período de março de 2005 a junho de 2006, 108 pacientes com diagnóstico de câncer de mama foram submetidas à cirurgia conservadora ou mastectomia com DA ou BLS. Não houve diferença estatisticamente significante entre os três grupos de estudo quanto às variáveis idade, estado civil, grau de instrução, status menopausal e lateralidade do tumor. Os tipos de tratamento e estadiamento encontram-se na Tabela I. A maioria das pacientes, 96 (88,9\%), fez tratamento adjuvante. 


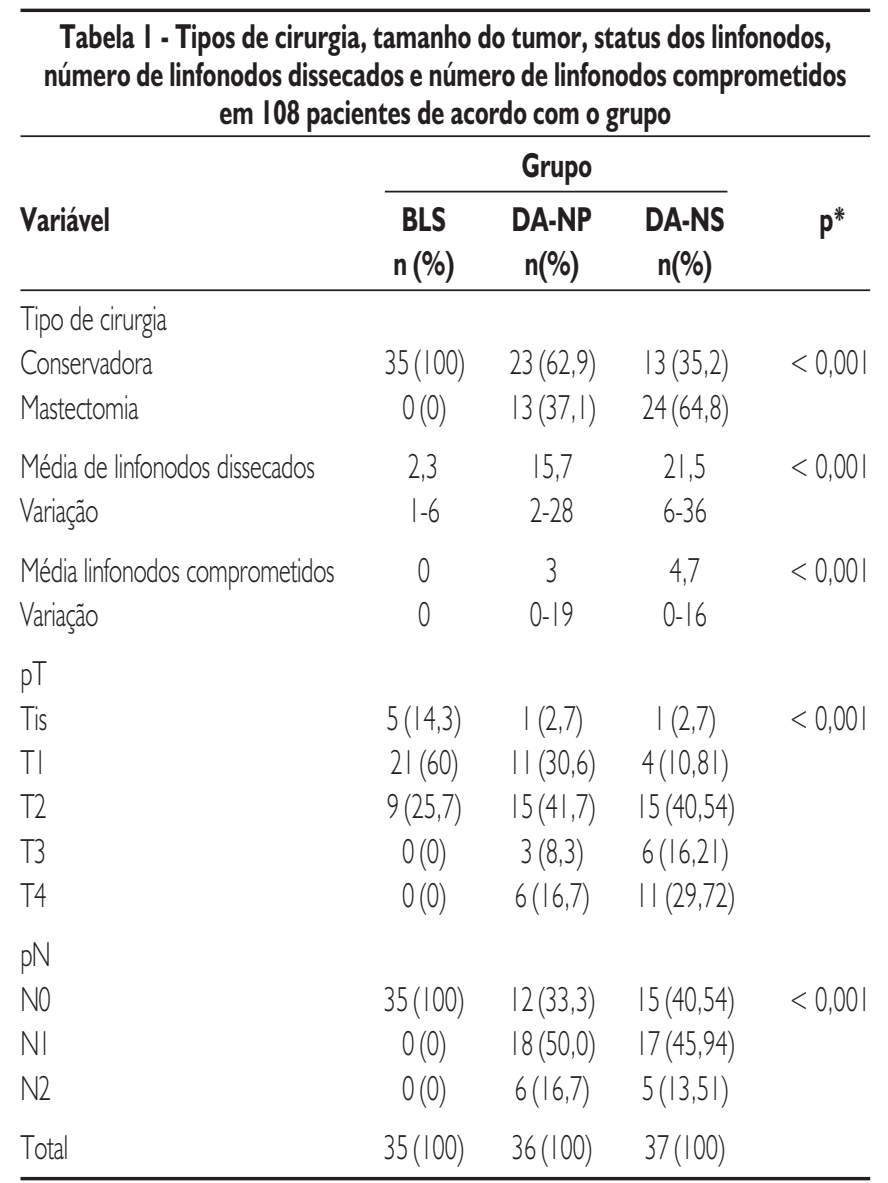

BLS: biópsia de linfonodo sentinela; DA-NP: dissecção axilar com nervo intercostobraquial preservado; DA-NS: dissecção axilar com nervo intercostobraquial seccionado; pT: tamanho tumoral no estadiamento patológico; pN: status dos linfonodos no estadiamento patológico * Teste ANOVA

Quimioterapia foi realizada em 59 (54,6\%) pacientes. Radioterapia foi feita em $96(88,9 \%)$ das pacientes e hormonioterapia foi administrada a $59(54,6 \%)$. Tratamentos adjuvantes associados foram empregados em 88 (81,5\%) pacientes. Radioterapia e quimioterapia foram feitas em $29(26,9 \%)$ pacientes, radioterapia e hormonioterapia em 33 (30,5\%) pacientes, quimioterapia e hormonioterapia em duas $(1,9)$ pacientes. Em 24 (22,2\%) pacientes foi presente a associação de radioterapia, quimioterapia e hormonioterapia. Seis pacientes não fizeram nenhum tipo de tratamento adjuvante.

Das 108 pacientes avaliadas, 45 pacientes $(41,7 \%)$ apresentaram pelo menos uma complicação pós-cirúrgica, imediata ou tardia (Tabela 2). A complicação mais comum foi dor, seguida de seroma, déficit sensorial, linfedema e infecção. Dor, linfedema e déficit sensorial foram mais freqüentes no grupo DA-NS. Houve diferença estatisticamente significante entre os três grupos somente quanto à presença de déficit sensorial $(p=0,04)$ (Tabela 2).

$\mathrm{Na}$ análise multivariada foram encontradas associações entre dor e infecção com risco relativo de 6, 19 vezes para as pacientes que tiveram infecção no pós-operatório (IC 95\% I,80-21,24; p=0,004). A presença de dor foi associada a $71 \%$ de chance de ter alteração de sensibilidade (IC 95\% 0, I2-0,73; p=0,009). Mulheres com idade abaixo de 45

\begin{tabular}{|c|c|c|c|c|}
\hline \multirow[b]{2}{*}{ Variável } & \multicolumn{3}{|c|}{ Grupo } & \multirow[b]{2}{*}{$\mathrm{p}^{*}$} \\
\hline & $\begin{array}{l}\text { BLS } \\
n(\%)\end{array}$ & $\begin{array}{c}\text { DA-NP } \\
\mathrm{n}(\%)\end{array}$ & $\begin{array}{c}\text { DA-NS } \\
\mathrm{n}(\%)\end{array}$ & \\
\hline \multicolumn{5}{|l|}{ Dor } \\
\hline Sim & || $(3 \mid, 4)$ & $12(33,3)$ & $14(37,8)$ & 0,77 \\
\hline Não & $24(68,6)$ & $24(66,7)$ & $23(62,2)$ & \\
\hline \multicolumn{5}{|l|}{ Seroma } \\
\hline Sim & $7(20)$ & $13(37,1)$ & $10(27)$ & 0,32 \\
\hline Não & $28(80)$ & $33(62,9)$ & $27(73)$ & \\
\hline \multicolumn{5}{|l|}{ Infecção } \\
\hline Sim & $4(1 \mid, 4)$ & $9(25)$ & $3(8,1)$ & 0,1 \\
\hline Não & $31(88,6)$ & $27(75)$ & $34(91,9)$ & \\
\hline \multicolumn{5}{|l|}{ Linfedema } \\
\hline Sim & $3(8,6)$ & $4(\mid I, I)$ & $10(27)$ & 0,06 \\
\hline Não & $32(91,4)$ & $32(88,9)$ & $27(73)$ & \\
\hline \multicolumn{5}{|c|}{ Déficit sensorial } \\
\hline Sim & $5(14,3)$ & $9(25)$ & $15(40,5)$ & 0,04 \\
\hline Não & $30(85,7)$ & $27(75)$ & $20(59,5)$ & \\
\hline Total & $35(100)$ & $36(100)$ & $37(100)$ & \\
\hline
\end{tabular}

BLS: biópsia de linfonodo sentinela; DA-NP: dissecção axilar com nervo intercostobraquial preservado; DA-NS: dissecção axilar com nervo intercostobraquial seccionado * Teste KruskalWallis

anos apresentaram 3,23 vezes chance de apresentarem dor (IC 95\% $1, \mid 3-9,21 ; p=0,028)$. Foi encontrada associação entre infecção e dor imediata com risco relativo de 6,43 (IC 95\% I,50-27,55 $p=0,0$ I2). Pacientes com seroma apresentaram risco relativo de 7,65 vezes de ter infecção (IC 95\% |,| 8-49,67|; $p=0,033$ ) do que as pacientes sem seroma. As pacientes do grupo BLS apresentaram chance $66 \%$ menor de ter déficit sensorial em relação aos grupos DA (IC 95\% 0, I I7-0,98; $p=0,047$ ). Não houve associação para a presença de linfedema.

No grupo BLS, a maioria das pacientes apresentou grau de incapacidade zero, apresentando sensibilidade aos monofilamentos $0,2 g$ (azul) e $2 g$ (violeta). Somente duas pacientes $(5,8 \%)$ do grupo BLS e quatro pacientes $(11,2 \%)$ do grupo DA-NP apresentaram grau de incapacidade um, apresentando sensibilidade aos monofilamentos de $4 \mathrm{~g}$ (vermelho) e $\log$ (laranja). No grupo DA-NS, 18 pacientes (50\%) apresentaram grau de incapacidade um. Sensibilidade ao monofilamento de $300 \mathrm{~g}$ (vermelho magenta), que significa incapacidade um, foi observada apenas em pacientes do grupo DA-NS. Uma paciente deste grupo não foi submetida ao teste devido à presença de linfedema severo. As diferenças foram estatisticamente significantes entre os três grupos para os testes com os monofilamentos de $0,02 \mathrm{~g}$ $(p<0,00 I), \log (p<0,004)$ e $300 g(p<0,00 I)$. 
Ferreira BPS et al.

\section{Discussão}

No presente estudo avaliamos a morbidade pós-cirúrgica em pacientes submetidas a BLS e DA com e sem preservação do nervo ICB. Estudos de outros autores compararam a morbidade associada aos dois procedimentos, sem relacionar a preservação do nervo $\mid \mathrm{CB}^{8,11}$.

Nossos resultados corroboram achados de estudo prévio de nosso grupo ${ }^{5}$ e de outros autores que a não preservação do nervo ICB está associada a maior déficit sensorial, ${ }^{3,1}$. Há poucos trabalhos na literatura que avaliaram a importância da preservação do nervo ICB durante a DA e eles mostraram resultados semelhantes aos nossos ${ }^{3,12}$.

A análise univariada mostrou que as pacientes dos grupos de BLS e DA-NP não apresentaram diferenças estatisticamente significantes na avaliação sensorial para nenhum dos monofilamentos de SemmesWeinstein. No entanto, quando comparamos com o grupo DA-NS, observamos diminuição da sensibilidade protetora até sua perda completa. O déficit sensorial foi mais freqüente neste grupo. Outros estudos também mostraram ser esta alteração mais comum, estando mais relacionado com a lesão do nervo ICB do que com a extensão da cirurgia axilar ${ }^{8,13}$

Neste trabalho, confirmamos que os monofilamentos de Semmes-Weistein podem aumentar a objetividade na avaliação sensorial e foram usados em estudo anterior de nosso grupo que sugeriu sua utilidade como ferramenta complementar na avaliação sensorial no câncer de mama ${ }^{5}$. Eles foram considerados, inicialmente, instrumentos de avaliação mais objetivos e confiáveis para avaliação sensorial em doenças neurológicas ${ }^{14}$.

No nosso estudo a presença de dor, de intensidade moderada ou grave, foi mais freqüente nas pacientes submetidas ao DA do que a BLS. No entanto, as diferenças não foram estatisticamente significantes. A presença de dor mesmo nos grupos BLS e DA-NP e com menor trauma cirúrgico sugere que o trauma cirúrgico e o processo de cicatrização também podem afetar a função do nervo. A presença de dor no grupo de DA-NS pode estar relacionada à possível formação de neuroma ou microneuromas 9,15 . No entanto, 23 pacientes $(62,2 \%)$ com DA-NS não apresentaram dor e 10 pacientes (27,05\%) não apresentaram déficit sensorial. É possível que nestas pacientes a inervação seja mantida devido às variações na anatomia e distribuição do suprimento nervoso da axila e braço'.

Com a evolução de cirurgias mais radicais para as cirurgias conservadoras, observou-se que dor crônica também ocorre após setorectomia ou quadrantectomia. O risco de lesão do nervo ICB que pode ocorrer durante DA é semelhante, tanto nas cirurgias conservadoras quanto nas mastectomias ${ }^{15}$. A ocorrência de dor no braço homolateral à cirurgia está mais relacionada à extensão do procedimento cirúrgico axilar do que ao tipo de cirurgia mamária, podendo estar associada a outros fatores como mostrado na análise multivariada.

A ocorrência de linfedema nos nossos grupos foi alta e variou de 8,6\% (BLS), II, I\% (DA-NP) e 27\% (DA-NS), porém sem diferença estatisticamente significante entre os três grupos, que em geral se manifesta mais tardiamente. Nossos dados estão de acordo com achados anteriores que relatam freqüência entre $7 \%$ e 30,5\% dos casos ${ }^{17,18}$. Não observamos relação estatisticamente significante entre o número de linfonodos dissecados, o número de linfonodos comprometidos e o desenvolvimento de linfedema.

$\mathrm{Na}$ ocorrência de seroma e infecção, não observamos diferença estatisticamente significante entre os grupos, embora estas alterações tenham ocorrido com maior freqüência nos dois grupos de pacientes submetidas a DA com e sem preservação do nervo ICB. O aparecimento de seroma e infecção têm sido associados a estadiamentos mais avançados e procedimentos mais radicais ${ }^{17}$.

Estudos mais recentes compararam a BLS com a DA e observaram que as complicações da DA são superestimadas, enquanto os aspectos negativos da BLS, subestimados ${ }^{19,20,21}$. A qualidade de vida depende de vários fatores somados ao tipo de abordagem axilar, no entanto, estudos prospectivos randomizados mostraram que pacientes submetidas a BLS obtiveram redução significante da morbidade física e psicológica com melhor função do braço e qualidade de vidaa 22,23 . As complicações estudadas foram observadas durante as avaliações das mulheres submetidas a BLS, mas com menor ocorrência em relação as mulheres submetidas a DA.

Mesmo sendo estes procedimentos realizados por cirurgiões experientes, recomenda-se que as pacientes sejam informadas sobre as possíveis complicações pós-cirúrgicas na BLS, assim como na DA $A^{17,24}$.

\section{CONCLUSÃo}

Nosso estudo demonstrou que a secção do nervo está relacionada a maior déficit sensorial havendo diferença estatisticamente significante entre os três grupos, o que não demonstrou ser significante com os demais critérios avaliados dentre os grupos analisados.

\section{Agradecimentos}

Agradeço especialmente às pacientes que aceitaram participar e contribuíram para este estudo e a Anke Bergmann e equipe pela disponibilidade e colaboração neste trabalho.

\section{Conflito de interesse: Não há}

\section{SUMMARY}

MORBIDITY AFTER SENTINEL NODE BIOPSY AND AXILLARY DISSECTION IN BREAST CANCER

BACKGROUND. The aim of this study was to evaluate the morbidity after sentine/node biopsy (SNB) andaxillary dissection with (AD-NS) or without sparing the intercostobrachial nerve (AD-NOS). Methods: A prospective cohort study was performed on 108 patients divided into three groups: $S N B(n=35), A D-N S(n=36)$ and $A D-N O S(n=37)$. We evaluated the incidence of sensory loss, pain, lymphedema, seroma formation and infection in the arm homolateral to the breast surgery. Semmes-Weinstein monofilaments were used to assess the sensoryloss; brachia/ perimetry was used to evaluate presence of lymphedema and a pain questionnaire was administered. ANOVA and Kruskal-Wallis statistical tests were used. Bivariate and Multivariate analyses were performed.

RESULTS. After surgery at least one complication was reported by 45/ 108 (41.7\%) patients. Pain was the outcome more often reported by patients. In the three groups a significant difference was observed only 
regarding sensory loss $(p=0.04)$. Pain, lymphedema, and sensory loss weremorefrequently found in the AD-NOS group. No significant difference was observed between SNB and AD-NS groups. Semmes-Weinstein monofilaments showed preservation of cutaneous sensitivity in 28/35 patients from the SNB group, in 25/36 patients from AD-NS group but in only 10/37 patients from $A D-N O S$ group $(p<0.001)$.

CONCLUSION. The ICBsection is associated with highersensoryloss, with statistically significant difference between the groups that were not shown to be significant with the others complications. [Rev Assoc Med Bras 2008; 54(6): 517-21]

KEY WORDS: Morbidity. Breast cancer. Sentinel node biopsy. Axillary dissection. Sparing. Intercostobrachial nerve.

\section{REFERÊNCIAS}

I. Luini A, Gatti G, Ballardini B, Zurrida S, Galimberti V, Veronesi P, et al. Development of axillary in breast cancer. Ann Oncol. 2005; | 6:259-62.

2. Giuliano AE, Jones RC, Brennan M, Stalman R. Sentinel lymphadenectomy in breast cancer. J Clin Oncol. 1997; 15:2345-50.

3. Torresan RZ, Santos CC, Conde DM, Brenelli HB. Preservação do nervo intercostobraquial na linfadenectomia axilar por carcinoma de mama. Rev Bras Ginecol Obstet. 2002;24:221-6.

4. Magaldi CM, Barros ACSD, Magaldi FM, Mantese JC, Pinotti JA. Avaliação da morbidade e funcionalidade do membro superior em mulheres submetidas à linfadenectomia axilar total e biópsia de linfonodo sentinela por câncer de mama. Rev Bras Mastol. 2005; I:9-14.

5. Pimentel MD, Santos LC, Gobbi H. Avaliação clínica da dor e sensibilidade cutânea de pacientes submetidas à dissecção axilar com preservação do nervo intercostobraquial para tratamento cirúrgico do câncer de mama. Rev Bras Ginecol Obstet. 2007;29:291-6.

6. Teicher I, Poulard B, Wise L. Preservation of the intercostobrachial nerve during axillary dissection for carcinoma of the breast. Surg Gynecol Obstet. | 982; | 55:89|-2.

7. Paredes JP, Puentes JL, Potel J. Variations in sensitivity after sectioning the intercostobrachial nerve. Am J Surg. 1990; 160:525-8.

8. Abdullah TI, Iddon J, Barr L, Baildam AD, Bundred NJ. Prospective randomized controlled trial of preservation of the intercostobrachial nerve during axillary node clearance for breast cancer. Br J Surg. 1998;85: I 4435.

9. Wallace MS, Wallace AM, Lee J, Dobke MK. Pain after breast surgery: a survey of 282 women. Pain. 1996;66: 195-205.

10. Ministério da Saúde. Fundação Nacional da Saúde. Guia de Controle da Hanseníase. Brasília; 1994.

I I. Veronesi U, Paganelli G, Viale G, Luini A, Zurrida S, Galimberti V, et al. A randomized comparison of sentinel-node biopsy with routine axillary dissection in breast cancer. N Engl J Med. 2003;349:546-53.
12. Taylor KO. Morbidity associated with axillary surgery for breast cancer. ANZ J Surg. 2004;74:3। 4-17.

13. Warmuth MA, Bowen G, Prosnitz LR, Chu L, Broadwater G, Peterson B, et al. Complications of axillary lymph node dissection for carcinoma of the breast: a report based on a patient survey. Cancer. 1998;83:1362-8.

14. Lehman LF, Orsini MBP, Nicholl ARJ. The development and adaptation of the Semmes-Weinstein monofilaments in Brazil. J Hand Ther. 1993;6:290-7.

15. Jung BF, Ahrendt GM, Oaklander AL, Dworkin RH. Neuropathic pain following breast cancer surgery: proposed classification and research update. Pain. 2003; 104:1-13.

16. Loukas M, Hullett J, Louis RG, Holdman S, Holdman D. The gross anatomy of the extrathoracic course of the intercostobrachial nerve. Clin Anat. 2006:19:106-11.

17. Wilke GL, McCall LM, Posther KE, Whitworth PW, Reintgen DS, Leich AM, et al. Surgical complications associated with sentinel lymph node biopsy: results from a prospective international cooperative group trial. Ann Surg Oncol. 2006; 13:49|-500.

18. Bergmann A. Prevalência de linfedema subsequente a tratamento cirúrgico para câncer de mama [dissertação]. Rio de Janeiro: Escola Nacional de Saúde Pública; 2000.

19. Silberman AW, McVay C, Cohen JS, Altura JF, Brackert RN, Sarna GP, et al. Comparative morbidity of axillary lymph node dissection and the sentinel lymph node technique: implication for patients with breast cancer. Ann Surg Oncol. 2004;240: I-6

20. Fleissig A, Fallowfield LJ, Langridge Cl, Johnson L, Newcombe RG, Dixon JM, et al. Post-operative arm morbidity and quality of life. Results of the ALMANAC randomized trial comparing sentinel node biopsy with standart axillary treatment in the management of patients with early breast cancer. Breast Cancer Res Treat. 2006;95:279-93

21. Mansel RE, Fallowfield L, Kissin M, Goyal A, Newcombe RG, Dixon JM, et al. Randomized multicenter trial of sentinel node biopsy versus standard axillary treatment in operable breast cancer: the ALMANAC trial. J Natl Cancer Inst, 2006;98:599-609

22. Purushotham AD, Upponi S, Klevesath MB, Bobrow L, Millar K, Myles JP, et al. Morbidity after sentinel lymph node biopsy in primary breast cancer: results from a randomized controlled trial. J Clin Oncol, 2005;23:43 I2-2I.

23. Mansfield L, Sosa I, Dionello R, Subramanian A, Devalia H, Mokbel K. Current management of the axila in patients with clinically node-negative câncer: a nationwide survey of United Kingdom breast surgeons. Int Semin Surg Oncol. 2007;4:4- 10.

24. Neumayer L, Meterissioan S, McMasters K. Canadian association of general surgeons and American college of surgeons evidence based reviews in surgery.23. ASCO recommended guidelines for sentinel lymph node biopsy for early-stage breast cancer. J Can Chir. 2007;50:482-4

Artigo recebido: 1//12/07

Aceito para publicação: 28/03/08 